\title{
Radiological abnormalities following bronchial thermoplasty: is the pathophysiology understood?
}

\author{
To the Editor:
}

We read with great interest the paper by DeBRAY et al. [1] reporting on early radiological lung abnormalities on computed tomography (CT) of the chest after bronchial thermoplasty (BT). The described findings in 13 patients are in line with our observations in 12 patients with severe asthma treated with BT in the TASMA trial (www.clinicaltrials.gov identifier number NCT02225392). Transient radiological abnormalities were seen after all 36 BT procedures, predominantly consisting of peribronchial consolidations with ground-glass opacities (figure 1a and c), partial occlusions/filling of bronchial lumen and atelectasis. Furthermore, we also observed a residual bronchial dilatation in a single case [2].

In contrast to the results of DeBRAY et al. [1], atelectasis directly after BT was less frequently observed in our patient cohort (38\% versus $68 \%$ of the BT procedures). We systematically scored endobronchial abnormalities before and immediately after BT by asking the bronchoscopist to score mucosal injury by grading for bronchial oedema, inflammatory aspect, secretions, mucus plugging and bleeding using a $0-3$ intensity score for each item (0: no abnormalities; 1 : minimal; 2 : moderate; 3 : severe intensity of abnormality). Grading was performed directly after the BT procedure by the interventional pulmonologist (J.T. Annema or P.I. Bonta). In the patients that were evaluated with a chest CT scan immediately after BT $(n=16)$, a significantly higher median mucosal injury score was seen in patients with atelectasis compared to patients without atelectasis (8 (interquartile range (IQR) 7-8) versus 6 (IQR 5-6.25) $(\mathrm{p}=0.0156)$, respectively). No significant difference was observed between the number of activations and the presence of atelectasis. Considering this, the observed difference in the occurrence of atelectasis directly after BT might be related to the vulnerability of the mucosa as is reflected by the endobronchial mucosal injury score.

We can confirm non-BT-treated lobe involvement as described by DeBray et al. [1], although we have not observed involvement of the non-BT-treated middle lobe. For the involvement of the non-BT-treated middle lobe, Debray et al. [1] provide two explanations: 1) diffusion of heat shock along the bronchial tree, which might be related to the earlier reported decrease in airway smooth muscle (ASM) area in the non-BT-treated middle lobe [3]; or 2) extension of heat shock through (incomplete) fissures to an adjacent lobe.

The first explanation seems unlikely since the decrease in ASM area in non-BT-treated middle lobe could not be confirmed by PRETOLANi et al. [4] investigating ASM decrease after BT in 15 patients. Furthermore, our results show that segmental/subsegmental airways that are not reached by BT but located adjacent and/or directly distal to BT-treated airways, showed no abnormalities on CT [2]. This observation makes the postulated explanation unlikely and suggest that radiofrequency energy is not diffused along the bronchial tree to other airways.

The second explanation, also supported by Boulet and LAVIOLETTE [5], seems a more plausible cause for the abnormalities seen in the non-BT-treated lobe as they were mostly found directly adjacent to incomplete fissures.

@ERSpublications

Radiological abnormalities after BT seems related to the severity of endobronchial mucosal injury after BT http://ow.ly/ZGeA30gLZa3

Cite this article as: d'Hooghe JNS, Bonta PI, van den Berk IAH, et al. Radiological abnormalities following bronchial thermoplasty: is the pathophysiology understood? Eur Respir J 2017; 50: 1701537 [https://doi.org/10.1183/13993003.01537-2017]. 

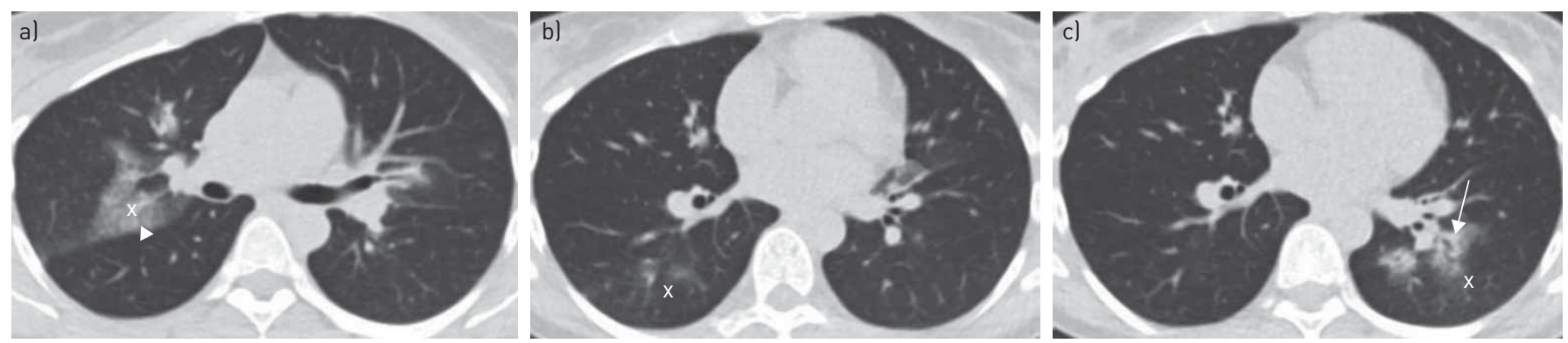

FIGURE 1 Radiological abnormalities seen in the bronchial thermoplasty (BT)-treated upper and non-BT-treated lower lobe. Ultra-low dose (ULD) chest computed tomography (CT) $<24 \mathrm{~h}$ after BT treatment of both upper lobes shows a) ground glass opacities (cross) restricted to the right upper lobe with a complete fissure (arrowhead) and b) ground glass opacities (cross) in a distal area of the non-BT-treated right lower lobe. These opacities were new since the ULD chest CT scan after the BT treatment of the left lower lobe 3 weeks earlier showed c) peribronchial consolidations (arrow) with ground glass opacities (cross) in the left lower lobe, but no abnormalities of the previously treated right lower lobe.

We postulate a third possible explanation for the radiological abnormalities observed in distal areas of the non-BT-treated lobes. The characteristics and distribution properties of these ground-glass opacities are compatible with blood, secretions and/or mucus (figure 1b). This could also explain why, in all of our cases, the radiological abnormalities were located in the non-BT-treated depending lower lobes only. Furthermore, this is in line with our observation that all cases with abnormalities observed in distal areas of the non-BT-treated lobes had a cumulative intensity score for mucosal bleeding, secretions and mucus after the BT procedure of $\geqslant 3$ and all the cases without involvement of the non-BT-treated lobes had a cumulative intensity score of 1 . Therefore, in our opinion, it is very likely that blood, secretions and mucus runs down from the BT-treated upper lobes to the depending lower lobes and causes the observed distal areas with ground glass opacities.

In conclusion, immediate radiological abnormalities following BT are common and transient. Direct and indirect radiofrequency energy-mediated pulmonary effects that can explain these radiological abnormalities are described. In addition, the occurrence of atelectasis in BT-treated lobes and the occurrence of ground-glass opacities in distal areas of non-BT-treated lobes seems related to the severity of endobronchial mucosal injury directly after BT.

Julia Nella Sofia d'Hooghe ${ }^{1}$, Peter Iwan Bonta ${ }^{1}$, Inge Alma Henrica van den Berk ${ }^{2}$ and Jouke Tabe Annema ${ }^{1}$ ${ }^{1}$ Dept of Respiratory, Academic Medical Center, University of Amsterdam, Amsterdam, the Netherlands. ${ }^{2}$ Dept of Radiology, Academic Medical Center, University of Amsterdam, Amsterdam, the Netherlands.

Correspondence: Jouke Tabe Annema, Dept of Respiratory Medicine, G5-247, Academic Medical Center, University of Amsterdam, PO Box 22700, NL-1100 DE, Amsterdam, The Netherlands. E-mail: j.t.annema@amc.nl

Received: May 232017 | Accepted: Aug 282017

Conflict of interest: Disclosures can be found alongside this article at erj.ersjournals.com

\section{References}

1 Debray MP, Dombret MC, Pretolani M, et al. Early computed tomography modifications following bronchial thermoplasty in patients with severe asthma. Eur Respir J 2017; 49: 1601565.

2 d'Hooghe JNS, van den Berk IAH, Annema JT, et al. Acute radiological abnormalities after bronchial thermoplasty: a prospective cohort trial. Respiration 2017; 94: 258-262.

3 Pretolani M, Dombret MC, Thabut G, et al. Reduction of airway smooth muscle mass by bronchial thermoplasty in patients with severe asthma. Am J Respir Crit Care Med 2014; 190: 1452-1454.

4 Pretolani M, Bergqvist A, Thabut G, et al. Effectiveness of bronchial thermoplasty in patients with severe refractory asthma: clinical and histopathological correlations. J Allergy Clin Immunol 2017; 139: 1176-1185.

5 Boulet LP, Laviolette M. Acute effects of bronchial thermoplasty: a matter of concern or an indicator of possible benefit to small airways? Eur Respir J 2017; 49: 1700029. 
From the authors:

We read with great interest the correspondence by J.N.S. d'Hooghe and colleagues focusing on the pathophysiology of early transient radiological abnormalities following bronchial thermoplasty (BT). In line with our previous report [1], they have reported transient radiological opacities in all treated lobes of 12 BT-treated patients and in an adjacent untreated lobe in $31 \%$ of cases [2].

Atelectasis observed in this study directly after BT was of lesser magnitude than that we have previously reported. This discrepancy may be related to the severity of the lobe volume loss taken into account. Indeed, we considered any lobe volume loss, including mild aerated lobar collapse manifesting solely as displacement of interlobar fissures, whereas J.N.S. d'Hooghe and colleagues might have only considered partly aerated lobe atelectasis.

The authors propose that endobronchial blood, secretions or mucus moving from the BT-treated upper lobes down to the lower lobes may be a novel explanation for the observed opacities in BT-untreated lobes. We agree with this hypothesis that is supported by their observations showing a higher score of endoscopic mucosal injury in patients with opacities in distal areas of the untreated lobes, as compared to cases without opacities. We did not perform any endobronchial mucosal injury scoring at the time of BT and, therefore, comparisons cannot be performed on this particular issue. However, we found opacities of nondependent lung zones in six out of 12 untreated lobes (middle lobe $(n=5)$ and culmen $(\mathrm{n}=1)$ ). Therefore, the hypothesis raised by J.N.S. d'Hooghe and colleagues seems unlikely to explain all untreated lobe opacities found in our study. In addition, the possibility that endobronchial secretions or mucus cause parenchymal ground-glass opacities appears questionable. Rather, we believe that this event may promote bronchial and bronchiolar obstruction, with subsequent atelectasis or centrilobular opacities.

J.N.S. d'Hooghe and colleagues also question our hypothesis that heat shock diffusion along the bronchial tree may explain such untreated-lobe opacities. Pretolani and co-workers [3, 4] have demonstrated histopathological effects of BT in untreated middle lung lobes, including in the airway smooth muscle (ASM) area and ASM-related nerves. These findings support the hypothesis that the effect of BT extends to nondirectly treated zones. Whether this would lead to parenchymal opacities remains unknown.

Finally, we agree with J.N.S. d'Hooghe and colleagues that more than one mechanism may cause early and transient opacities in non-BT-treated zones.

@ERSpublications

More than one mechanism may cause early and transient opacities in non-BT-treated lung zones http://ow.ly/3nfB30gzEpS

Cite this article as: Debray M-P, Dombret M-C, Pretolani M, et al. Radiological abnormalities following bronchial thermoplasty: is the pathophysiology understood? Eur Respir J 2017; 50: 1702067 [https://doi. org/10.1183/13993003.02067-2017].

Marie-Pierre Debray ${ }^{1}$, Marie-Christine Dombret ${ }^{2}$, Marina Pretolani ${ }^{3,4,5}$, Gabriel Thabut ${ }^{3,4,5,6}$, Loubna Alavoine ${ }^{7}$, Pierre-Yves Brillet $^{8,9}$, Camille Taillé, ${ }^{2,3,4,5}$, Antoine Khalil ${ }^{1,4}$, Pascal Chanez ${ }^{10}$ and Michel Aubier ${ }^{2,3,4,5}$

${ }^{1}$ Dept of Radiology, Bichat Claude Bernard Hospital, Assistance Publique-Hôpitaux de Paris, Paris, France. ${ }^{2}$ Dept of Pneumology A, Bichat Claude Bernard Hospital, Assistance Publique-Hôpitaux de Paris, Paris, France. ${ }^{3}$ Inserm UMR1152, Physiopathology and Epidemiology of Respiratory Diseases, Paris, France. ${ }^{4}$ Paris Diderot University, Faculty of Medicine, Paris, France. ${ }^{5}$ Laboratory of Excellence, INFLAMEX, Université Sorbonne Paris Cité and DHU FIRE, Paris, France. ${ }^{6}$ Dept of Pneumology B, Bichat Claude Bernard Hospital, Assistance Publique-Hôpitaux de Paris, Paris, France. ${ }^{7}$ Clinical Investigation Center, Bichat-Claude Bernard University Hospital, Paris, France. ${ }^{8}$ Dept of Radiology, Avicenne Hospital, Assistance Publique-Hôpitaux de Paris, Bobigny, France. ${ }^{9}$ Université Paris 13, PRES Sorbonne-ParisCité, UPRES EA 2363, Bobigny, France. ${ }^{10}$ Inserm U1067 and CNRS UMR7733, Dept of Respiratory Diseases, AixMarseille University, Marseille, France.

Correspondence: Marie-Pierre Debray, Dept of Radiology, Bichat Claude Bernard Hospital, Assistance PubliqueHôpitaux de Paris, 46 rue Henri Huchard, Paris, Ile de France 75018, France. E-mail: marie-pierre.debray@aphp.fr

Received: Oct 082017 | Accepted: Oct 092017

Conflict of interest: Disclosures can be found alongside this article at erj.ersjournals.com 


\section{References}

1 Debray MP, Dombret MC, Pretolani M, et al. Early computed tomography modifications following bronchial thermoplasty in patients with severe asthma. Eur Respir J 2017; 49: 1601565.

2 d'Hooghe JNS, van den Berk IAH, Annema JT, et al. Acute radiological abnormalities after bronchial thermoplasty: a prospective cohort trial. Respiration 2017; 94: 258-262.

3 Pretolani M, Dombret MC, Thabut G, et al. Reduction of airway smooth muscle mass by bronchial thermoplasty in patients with severe asthma. Am J Crit Care Med 2014; 190: 1452-1454.

4 Pretolani M, Bergqvist A, Thabut G, et al. Effectiveness of bronchial thermoplasty in patients with severe refractory asthma: clinical and histopathologic correlations. J Allergy Clin Immunol 2017; 139: 1176-1185.

Copyright (CERS 2017 